\title{
Design and Implementation of Agricultural Intelligent Monitoring System based on the Android Platform
}

\author{
Liang Haili ${ }^{1, a}$ \\ ${ }^{1}$ Department of Information Engineering, Weihai Vocational College, Weihai 264210,China \\ alianghaili@126.com
}

Keywords: Intelligent agriculture; Mobile Internet; HTTP communication; Android

\begin{abstract}
Relies on an intelligent agricultural experiment platform, designed and developed an intelligent agricultural monitoring system based on the Android system, making mobile Internet technology applied to traditional agriculture, allowing growers monitor environmental factors in agricultural production through mobile network anytime, anywhere, including air temperature and humidity, soil moisture, etc.. And allow the farmers set up the environment index threshold according to the need of agricultural production, if the environmental indicators more than the normal range, the system can automatically open water pump, fan and so on to improve the environment to avoid the adverse effects of agricultural production environment, thus realize the agricultural production environment automatic control, so as to achieve the goal of scientific technology. The system structure, the design of the server and client implementations are introduced in detail in this paper, as well as integrated use of the system design Zigbee communication key technology, the Internet of things, Android. The designed system in the experimental platform runs well.
\end{abstract}

\section{Introduction}

Agricultural production is closely related with national economy and people's livelihood, which is the foundation of social stability. As the national "Internet + " policy introduced, the high-speed development of modern agriculture in China, the demand for agricultural production and management of the scientific, intelligent is more and more intense. It is well known that environmental climate has been the main factor affecting the agricultural production, especially to the influence of heat, water, light, is the largest. The emergence of mobile Internet technology makes remote control to become a reality, agricultural production personnel, able to monitor and control agricultural production environment via mobile Internet, namely the intelligent control of agriculture.

At present, both foreign countries and China have carried out a similar study in the field of intelligent agriculture [1], mainly divided into two aspects, one is the agricultural products supply and marketing system, similar to the electronic shopping mall and primarily for agricultural products and agricultural materials supply and marketing to build a platform; On the other hand is the monitoring of crop production index [2]. No matter what is in the "Internet + " environment, it is good for intelligent agricultural system. Intelligent agricultural system designed in this paper takes intelligent agriculture on the test bench, simulate the real environment of agricultural production, and verify the reliability of the system is running. In system development, the integrated use of the wireless communication, mobile Internet, Android and other technology, realized the agriculture 
field environment remote monitoring, early warning and control, and be able to analyze the environmental factors, so as to guide the agricultural production.

\section{The system architecture design}

Intelligent agricultural system designed in this paper consists of three parts, one is wireless sensor network made up of many sensors and control unit, the second is server that can provide the available sensor data, communications, and public service, the third is the Android platform of mobile client [3]. In this paper, the author cooperates with enterprise in setting up common structures, intelligent agriculture simulation test bench. The system of the design and development, are based on the test bench, the relationship between each part is shown in figure 1 .

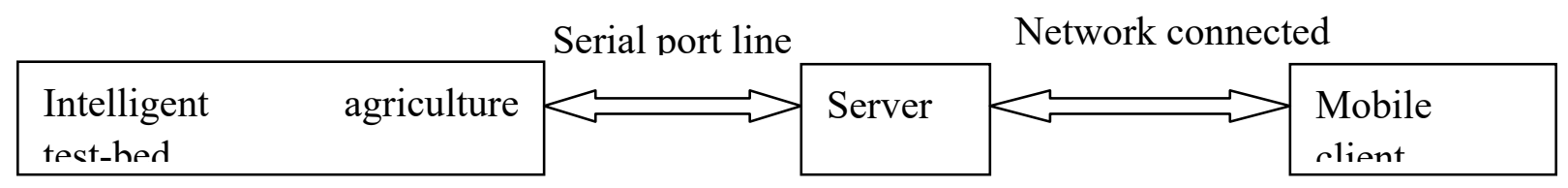

Figure 1. Intelligent agricultural system topology

\section{The system main function module}

Main function modules of the system are environmental monitoring, threshold Settings, and remote control of three modules, including environmental monitoring module to monitor air temperature, humidity, illumination in the agricultural production, soil moisture; Threshold setting module can set of agricultural production in the most suitable air temperature, humidity, illumination, soil humidity range; Remote control module allows users to remote control equipment of open control system.

System mainly has two data flow, as shown in figure 2. The first collect agricultural field environment information at any time, including air temperature and humidity, soil moisture, etc., through the sensor, and then through the server side, pass the data to the mobile client, according to needs to be saved to the database; the second is the user make good decisions according to the detected data or the results of data analysis, sending control command, through the server to the system control unit, control of agriculture greenhouse irrigation control, ventilation, lighting and other equipment, to ensure that the growth of crops is always in a good environment.

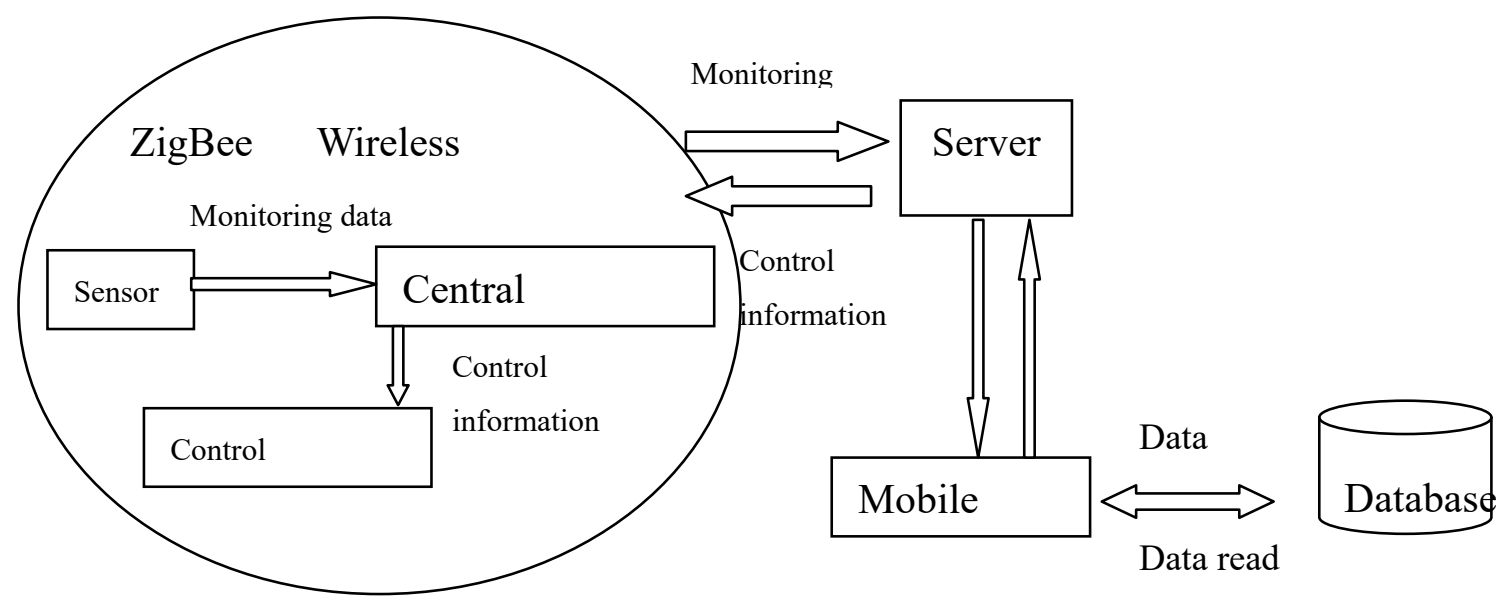

Fig 2. Data flow diagram of system 


\section{Design and implementation of system main function module}

\subsection{Wireless sensor network design}

The intelligent agricultural experiment platform this paper use is designed by the enterprise, on which basis the author completed the development of software system design. Intelligent agricultural experiment station design a number of sensor nodes, and has formed a wireless sensor network through the Zigbee agreement, each sensor node through the central coordinator (single-chip microcomputer) exchange of data. In order to guarantee the accuracy of the data communication, the central coordinator exchange data on the server through RS232 serial port cable and intelligent [4]. Wireless sensor network provides read data command and control interface, for the server-side call.

\subsection{The server side design}

In this system, the server is a data transmission hub. When install the Android platform, use free, lightweight NanoHTTPD as the HTTP server, when the server starts up, need to obtain real-time intelligent agricultural experiment station data, read serial data in the Service, when the server starts up, the Service is started, run in the background, real-time access to serial data. Server side loop read serial data using the Timer class implements [5], the main code is as follows:

timer $=$ new timer () ;

timer. schedule (new TimerTask ()$\{/ /$ call the schedule method, create a thread public void the run () \{

. / / call the wireless sensor network provides interfaces, read sensor data

$$
\}
$$

\}, 1000, 1000); / / every two seconds for the sensor data

In system design and development, the server and the client using wi-fi network, using the HTTP protocol, through the IP address and a serial port communication, data transmission using lightweight, and relatively simple JSON format [6]. The server to obtain IP address of the main code is as follows:

WifiManager WifiManager $=($ WifiManager $)$ getSystemService (WIFI_SERVICE);

WifiInfo WifiInfo = wifiManager. GetConnectionInfo ();

Int ipAddress $=$ wifiInfo. GetIpAddress (); / / get the IP address of the WIFI

String IP $=($ ipAddress \& 0 XFF $)+" "+(($ ipAddress $>>8) \& 0$ XFF $)+"$ "+ ((ipAddress $>>16) \&$ 0 XFF $)+"$ " + ((ipAddress $>>24) \& 0$ XFF $) ; / /$ IP address translation

\subsection{The mobile client design}

Mobile client uses the Android platform get each sensor data from the server through the wireless network, based on the HTTP protocol to, and to save data to a local database. It can conduct analysis of the corresponding data according to user needs to query the historical data, it can also set the range of each sensor monitoring, as the basis of a system for early warning. At the same time the system provide automatic or manual open control unit in two ways, convenient user according to the detected data, and timely adjustment of agricultural production environment.

In order to be able to clearly observe the sensor data, the client uses the line chart shows that using thread every two seconds to read a data, various sensors according to use sliding screen switch, fragments techniques are used to the client shows mainly, through threads access to server data, using the Handler to update the user interface [5], in order to get the air temperature as an example, the main code is as follows:

public class chart1 extends fragments $\{/ /$ Fragement class inheritance

private ChartView chart1; / custom View subclass

private Timer Timer; 
private Handler Handler.

public View onCreateView (LayoutInflater inflater, @ Nullable ViewGroup container, @ Nullable Bundle savedInstanceState) \{ view the View $=$ inflater. Inflate (R.1 ayout. Chart1, container, false);

Init (view);

return the view;

\}

private void init (View View) \{

chart $1=($ ChartView $)$ view. The findViewById (R.i, dc hart1);

timer $=$ new timer ()$; / /$ timer

timer. The schedule (new TimerTask () $\{/ /$ create a thread

public void the run () \{

Message MSG = new Message (); / / create the Message object, used to carry data

msg. what = MainActivity. Current_airtemper; / / the air temperature in the Message

object

handler. SendMessage (MSG); / / the Handler object to send the Message object to the main thread

\}

\}, 1000, 1000);

handler $=$ new handler ()$\{/ /$ create a handler object, and used to update the interface

public void handleMessage (Message MSG) \{

super. HandleMessage (MSG);

chart1. Update (MSG. What); / / updated in real time data and redraw interface

\}

\}$;\}\}$

\section{The system operation}

This paper uses of "Internet + traditional agriculture" model, designed an intelligent agricultural environment monitoring system. The experiment platform run well, this can effectively monitor simulation experiment platform of environmental factors in agricultural production.

System main interface is shown in figure 3 to figure 4. Figure 3 is sensor historical data query and analysis of interface, the user can choose the sensors, and select the data query, analysis, the time period; Data analysis can bar charts and pie charts form; Figure 4 shows the control unit current state, the user can also through the bottom of the button control, if close the manual control mode, the system will according to the corresponding numerical, sensors to detect more than warning or resume normal, automatic open or close the control unit.

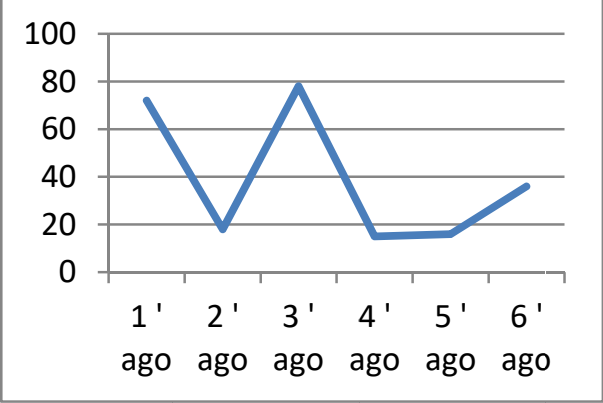

Figure 3. Query and analysis of sensor historical data

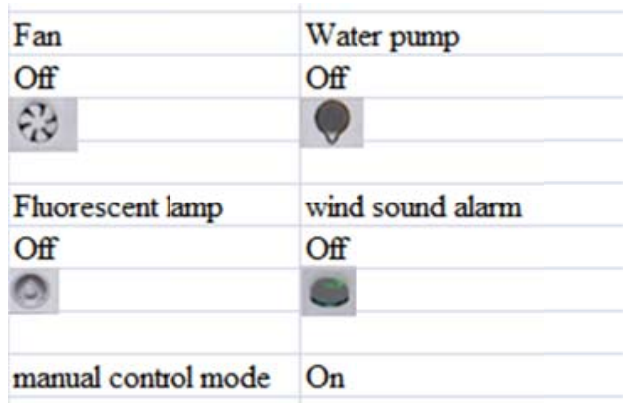

Figure 4. Open or close the control unit 


\section{Summary}

With the popularity of smart devices, mobile Internet has entered innumberable families. The concept of intelligent agricultural environment monitoring system designed in this paper is based on the Android platform of mobile Internet system, which can realize real-time adjustment for the environment of agricultural production in abnormal situation and avoide what shall circumvent the risk of agricultural production, thus effectively reduce the agricultural production cost, and disruptive change for agricultural production. The design of environmental monitoring, early warning system are based on intelligent agricultural experiment station, but the software system design technology used can be applied to other areas, such as the application in air environmental monitoring or production environment monitoring system. The application of mobile Internet technology in agricultural production in this paper research has made the exploration effectively.

\section{References}

[1] Geng Juntao, Zhou Xiaojia, Zhang Bingjie. The atmospheric environment monitoring system based on wireless sensor network design [J]. Journal of xihua university (natural science edition), 2007, (4) 44-46

[2] LIN Yuanguai, XIE Haixia, et al. The Design of Zigbee Gateway in Intelligent Monitoring System for Agriculture [C]. 2011 International Conference on Mechatronic Science, Electric Engineering and Computer. The IEEE Computer Society, 2011:2213-2216

[3] $\mathrm{Xu}$ Wei. Based on the Internet of things intelligent energy-saving and intelligent transportation key technology research and application [D], Nanjing: Nanjing University of posts and telecommunications, 2013:14-16

[4] ATZORI Luigi, IERA Antonio, MORABITO Giacomo. The Internet of Things: A survey [R]. Computer Networks, 2010, (15) : 2787-2805

[5] Li gang. Crazy Android notes (third edition) [M]. Beijing: electronic industry press, 2015:156-168

[6] Li Xinghua. Android development practical oral [M]. Beijing: Tsinghua University press, 2012:643-646 